11

\section{BRONCHOALVEOLAR LAVAGE FLUID CYTOKINE AND CHEMOKINE PROFILES IN VENTILATED PRETERM INFANTS}

J.V. Been ${ }^{1}$, J.F. van Iwaarden', W. de Jager², A. Debeer ${ }^{3}$, L.J. Zimmermann ${ }^{1}$

${ }^{1}$ Paediatrics, Maastricht University Medical Centre, Maastricht, ${ }^{2}$ Paediatric Immunology, University Medical Center Utrecht, Utrecht, The Netherlands, ${ }^{3}$ Neonatology, University Hospital "Gasthuisberg, Leuven, Belgium

Background and aims: Pulmonary inflammation is historically regarded a key element of the pathophysiology of bronchopulmonary dysplasia (BPD). We aimed to determine the temporal cytokine and chemokine patterns in bronchoalveolar lavage fluid (BALF) from ventilated pretem infants in the first postnatal week. Associations with exposure to chorioamnionitis and development of BPD were determined.

Methods: BALF was collected in ventilated preterm infants ( $\leq 32 \mathrm{wks}$ ) on days $0,1,3$ and 7 . Using multiplex immunoassay, individual levels of 32 inflammatory mediators were quantified in each sample.

Results: 93 BALF samples were obtained from 59 infants. Levels were within the detection range for the vast majority of mediators and samples. Histological chorioamnionitis was associated with significantly increased BALF cytokine and chemokine levels, most prominently so on day 0 (Figure 1A). IL-10, TNF- $\alpha$, IL-1 $\alpha$, and IL-1 $\beta$ had the highest predictive ability for chorioamnionitis on day 0 (area under ROC curve $>0.90 ; p<.01$ ). Increased cytokine and chemokine levels were not predictive of BPD development (Figure 1B). In contrast, BPD was associated with significantly decreased levels of several chemokines during the first postnatal week (CCL5, -19 and -22, CXCL8, and MIF (all $\mathrm{p}<.05)$.
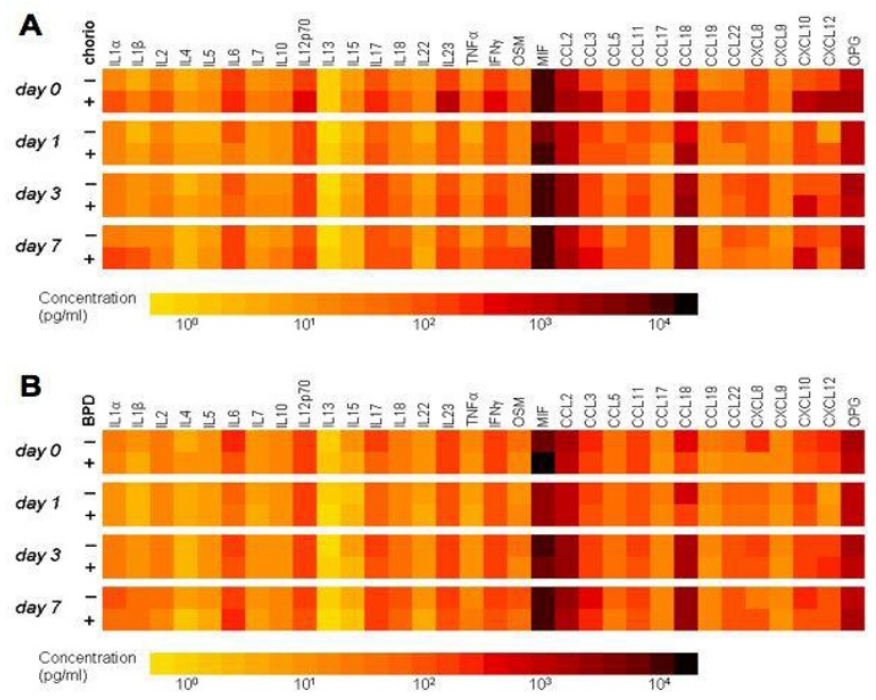

Figure 1. Bronchoalveolar lavage fluid cytokine and chemokine profiles according to antenatal exposure to chorioamnionitis $(A)$ or subsequent development of BPD (B)

\section{[Figure 1]}

Conclusions: BPD was not preceded by increased pulmonary levels of inflammatory mediators during the first postnatal week in preterm infants. This is in accordance with the concept of 'new BPD' characterised by lung developmental arrest rather than lung injury and inflammation.

12

\section{HYPERTENSION, DIABETES AND OVERWEIGHT: LOOMING LEGACIES OF THE BIAFRAN FAMINE}

M. Hult ${ }^{1}$, P. Tornhammar ${ }^{1}$, P. Ueda ${ }^{1}$, C. Chima ${ }^{2}$, A.-K. Edstedt Bonamy ${ }^{3}$, B. Ozumba ${ }^{2}$, M. Norman ${ }^{1}$

${ }^{1}$ Department of Clinical Science, Intervention and Technology (CLINTEC), Karolinska Institute,

Stockholm, Sweden, ${ }^{2}$ University of Nigeria Teaching Hospital, Enugu, Nigeria, ${ }^{3}$ Department of Woman and Child Health, Karolinska Institute, Stockholm, Sweden

Objective: To study the risks for hypertension, glucose intolerance and overweight forty years after fetal or childhood exposure to famine afflicting Biafra during the Nigerian civil war (1967-1970).

Design: Prospective cohort study performed in June 27 - July 31, 2009.

Setting: Enugu, Nigeria.Participants: Adults $(n=1,339)$ born before (1965-67), during (1968-January 1970), or after (1971-73) the years of famine. 
Main outcome measures: Blood pressure (BP), random plasma glucose and anthropometrics, as well as prevalence of hypertension (BP >140/90 $\mathrm{mmHg}$ ), impaired glucose tolerance (IGT; p-glucose 7.8-11.0 $\mathrm{mmol} / \mathrm{l}$ ), diabetes (DM; p-glucose $\geq 11.1$ $\mathrm{mmol} / \mathrm{l})$, or overweight $\left(\mathrm{BMI}>25 \mathrm{~kg} / \mathrm{m}^{2}\right)$.

Results: Fetal-infant exposure to famine was associated with elevated systolic $(+7 \mathrm{mmHg}$; $\mathrm{p}<$ $0.001)$ and diastolic $(+5 \mathrm{mmHg} ; p<0.001) \mathrm{BP}$, increased $p$-glucose $(+0.3 \mathrm{mmol} / \mathrm{L} ; \mathrm{p}<0.04)$ and waist circumference $(+3 \mathrm{~cm}, p<0.001)$, increased risk of systolic hypertension (adjusted OR 2.87; $95 \% \mathrm{Cl} 1.90-4.34$ ), IGT (OR 1.65; 95\% Cl 1.022.69) and overweight (OR 1.41; 95\% Cl 1.03-1.93) as compared to people born after the famine. The effect of fetal-infant famine on BP was seen in both women and men, whereas the effect on IGT was seen in men only, and the increased risk of overweight was confined to women.

Conclusions: Fetal and infant undernutrition is associated with significantly increased risk of hypertension and impaired glucose tolerance in 40-year-old Nigerians. Prevention of undernutrition during pregnancy and in infancy should therefore be given high priority in health, education, and economic agendas.

\section{3}

DOES FOLIC ACID INTAKE IN PREGNANCY INCREASE RISK OF WHEEZE AT 18 MONTHS? EVIDENCE FROM THE DANISH NATIONAL BIRTH COHORT.

\author{
E. Maslova' ${ }^{1}$, C. Granstrom², S. Petersen², \\ E. Oken ${ }^{3}$, S.F. Olsen ${ }^{2}$
}

${ }^{1}$ Nutrition, Harvard School of Public Health, Boston, MA, USA, ${ }^{2}$ Maternal Nutrition Group, Danish Epidemiology Science Centre, Statens Serum Institut, Copenhagen, Denmark, ${ }^{3}$ Obesity Prevention Program, Department of Population Medicine, Harvard Medical School and Harvard Pilgrim Health Care Institute, Boston, MA, USA

Background: Recent evidence suggests that maternal supplementation of folic acid during pregnancy may play a role in the development of wheezing and asthma among offspring, potentially through epigenetic mechanisms.

Objective: We examined associations of maternal prenatal intake of folic acid supplements and dietary folate with wheeze and recurrent wheeze among offspring at age 18 months in the Danish National Birth Cohort.

Methods: We estimated maternal intake of folic acid via supplements and dietary folate $(n=54,440)$ using a validated FFQ completed in mid-pregnancy. We defined wheeze as a positive maternal report of child wheezing on either the 6 or 18 month interview, and recurrent wheeze as $\geq 3$ wheeze episodes with the first occurring before 15 months of life. We conducted logistic regression analyses controlling for potential confounders, and report here estimates for the highest vs. lowest quartiles.

Results: Of the 54,440 children, $14.2 \%(n=7,703)$ had wheezed and $3.0 \%(n=1,619)$ had recurrent wheezing by 18 months. Maternal intake of folic acid supplements was not associated with child wheezing (OR: $1.06,95 \% \mathrm{Cl}: 0.93,1.21)$ or recurrent wheezing. (OR: $1.13,95 \% \mathrm{Cl}: 0.88,1.46)$ Similarly, dietary folate was not associated with wheezing (OR: $0.95,95 \% \mathrm{Cl}: 0.87,1.04$ ) or with recurrent wheeze (OR: $0.90 ; 95 \% \mathrm{Cl}: 0.74,1.08)$.

Conclusions: We found no association of maternal mid-pregnancy intake of folic acid supplements or dietary folate with wheezing in early childhood. Maternal folic acid status at conception may be more important in influencing child epigenetic imprinting and subsequent outcomes.

\section{4}

\section{METHIONINE REQUIREMENT IN PRESENCE OF CYSTEINE IN THE ENTERALLY FED TERM NEONATE}

\author{
L. Huang ${ }^{1}$, J.E. Schoonenboom ${ }^{1}$, \\ M. van Dongen ${ }^{1}$, F. Maingay-de Groof ${ }^{1}$, \\ G.J. Voortman', C. Chen², \\ Y. Huang ${ }^{3}$, J.B. van Goudoever ${ }^{1}$
}

${ }^{1}$ Neonatology, Erasmus MC - Sophia Children's Hospital, Rotterdam, The Netherlands, ${ }^{2}$ Neonatology, ${ }^{3}$ Gastroenterology, Children Hospital of Fudan University, Shanghai, China

Background: Experimental evidence of essential amino acid requirement in neonates is scanty. Recently, the branched chain amino acid requirements in term neonates were successfully determined using the indicator amino acid oxidation method. Methionine, an essential amino acid, can be used for protein synthesis, but serves as a precursor for homocysteine and cysteine as well. Current recommended total sulphur amino acid requirement for infants $0-1$ months is $57 \mathrm{mg} / \mathrm{kg} / \mathrm{d}$ (methionine 